Surface Review and Letters, Vol. 13, Nos. 2 \& 3 (2006) 265-271

(c) World Scientific Publishing Company

\title{
ADHESION OF PET/PSMA INTERFACES REINFORCED WITH PLASMA TREATMENT
}

\author{
CHI-AN DAI ${ }^{*, \ddagger}$, TAI-AN TSUI* and YAO-YI CHENG ${ }^{\dagger}$ \\ ${ }^{*}$ Department of Chemical Engineering and \\ Institute of Polymer Science and Engineering, \\ National Taiwan University, Taipei, 106 Taiwan, R.O.C \\ $\dagger$ Institute of Organic and Polymeric Materials, \\ National Taipei University of Technology, \\ Taipei, 106 Taiwan, R.O.C \\ †polymer@ntu.edu.tw
}

\begin{abstract}
The interface between biaxially oriented poly (ethylene terephthalate) (PET) films and poly (styrene-co-maleic anhydride) (PSMA) was reinforced by nitrogen plasma treatment of PET film and subsequent annealing treatment of the PET/PSMA bi-material. The fracture toughness, Gc, of the interface was quantitatively measured using an asymmetric double cantilever beam test (ADCB). X-ray photoelectron spectrometry (XPS) was used to measure the change in the surface composition of PET films upon plasma treatment and correlate the fracture toughness of the interface. The fracture energy of PET/PSMA interface is significantly enhanced by annealing the plasma treated PET with PSMA at a temperature greater than the glass transition temperature of PSMA $\left(\sim 120^{\circ} \mathrm{C}\right)$. At an annealing temperature of $150^{\circ} \mathrm{C}$, Gc increases with increasing plasma treatment time and reaches a plateau value of $\sim 100-120 \mathrm{~J} / \mathrm{m}^{2}$, a two order of magnitude increase in Gc compared with that of samples annealed at $130^{\circ} \mathrm{C}$. The enhancement of the adhesion is resulted from the in-situ formation of copolymers due to reaction between amine functional groups from the plasma treatment and anhydride groups from PSMA. For plasma treatment time $<10 \mathrm{~s}$, scanning electron microscope (SEM) measurement show that the fracture surface is relatively smooth indicating an interfacial failure between PET/PSMA. With increasing plasma treatment time and therefore increasing the amount of nitrogen functional groups on PET surface, large plastic deformation takes place at the PET/PSMA interface. For treatment time $\geq 100-150 \mathrm{~s}$, the PET/PSMA interface becomes stronger than PET bulk material and consequently crack deviates from the interface and the failure occurs within the PET film. The interlayer fracture energy of a biaxially oriented PET film can thus be quantitatively measured with a Gc value of roughly $120 \mathrm{~J} / \mathrm{m}^{2}$.
\end{abstract}

Keywords: Fracture toughness; plasma treatment; poly (ethylene terephthalate) (PET).

\section{Introduction}

Poly (ethylene terephthalate) (PET) is one of the most important classes of thermoplastic materials used as film, fiber and bottle. ${ }^{1,2}$ Because of its superb properties including thermal property, mechanical property, transparency, storage stability, gas barrier and inertness toward chemicals, PET is used as a leading support material for applications in coating, packaging, electronic, and biomedical industries. ${ }^{3}$ For example, PET has been used as a substrate material for artificial vessels. ${ }^{4}$ In order to improve the biocompatibility of PET substrate, surface

${ }^{\ddagger}$ Corresponding author. 
treatments or coatings are needed. ${ }^{5}$ However, the interface between biaxially oriented PET film and coatings is rather weak because of high crystallinity and its inertness toward chemicals of biaxially oriented PET films. Often a small excess force can cause delamination between coated layers and PET, which could severely affect the performance of the device in service. Therefore, finding a solution to improve the interfacial adhesion between two incompatible polymers has long been a critical issue and an actively researched topic. Moreover, many traditional methods to measure interfacial adhesion between two bulk materials, e.g., peel test, wedge test etc. give only qualitative information on adhesion. Therefore, there are great demands to explore new methods of measuring thin film adhesion.

Commercial PET flat films are formed by melt extrusion, biaxial drawing and heating treatment processes. During the drawing and heating processes, substantial increase in crystallinity and preferential chain orientation parallel to the film surface were resulted, which leads to anisotropy in its mechanical property. ${ }^{6,7}$ It is well known that if the crystals are too highly order parallel to the surface with too few tie-molecules binding them in the bulk, the surface crystal may delaminate under stress. ${ }^{8}$ However, a quantitative failure criterion for interlayer delamination was not previously established. In addition, although large body of literature in the past has studied the effect of plasma treatment on the surface composition change of many commercial plastic films,${ }^{9-14}$ direct relationship between the surface chemistry and fracture energy has not been fully exploited. The object of this study is to establish such relationship by using a quantitative adhesion measurement method.

\section{Experimental}

\subsection{Materials}

The $100 \mu \mathrm{m}$-thick poly (ethylene terephthalate) (PET) films used in this study were biaxially drawn untreated PET film purchased from the Shin-Kong Synthetic Fibers Corporation, Taiwan. Prior to plasma treatment, the PET film was cleaned by rinsing with distilled water and methanol and then in refluxing hexane for $2 \mathrm{~h}$. Poly (styrene-co-maleic anhydride) (PSMA) is a random copolymer with
$93 \mathrm{wt} \%$ of styrene and $7 \mathrm{wt} \%$ of anhydride units purchased from Aldrich Chemical Company. The weightaverage molecular weight of PSMA is $\sim 220000$ as measured from gel permeation chromatography (GPC) and its glass transition temperature is $\sim 120^{\circ} \mathrm{C}$ as measured from differential scanning calorimetry.

\subsection{Plasma treatment of PET}

Because of high crystallinity $(\sim 40-50 \%)$ of the PET film, the surface of PET film is generally difficult to adhere to without additional primer coating or energetic surface treatments. Plasma treatment or so-called glow discharge treatment (GDT) is known to enhance the wettability of the treated surface, to produce reactive functional groups, and to improve the adhesion between coated layers. ${ }^{13}$ Unless otherwise noted, all PET films were plasma treated using a radio-frequency $(13.56 \mathrm{MHz})$ power source and a matching impedance unit at a nitrogen pressure of $150 \mathrm{~m}$ Torr and an output power of $50 \mathrm{~W}$. We varied the duration of the plasma treatment from 0 to $400 \mathrm{~s}$ to enhance the amount of nitrogen incorporated on PET surface. The high voltage electrode was fabricated from titanium metal and the ground aluminum electrode is the vacuum chamber itself. PET film samples were placed on a sample plate, which was located between the high voltage electrode and the ground electrode. The electrode and sample plate geometry provides greater uniformity of the treated surface of samples as verified by the contact angle measurement.

\subsection{Surface analysis}

Once the PET film is plasma treated, the film is quickly transferred into the chamber of X-ray photoelectron spectroscopy (XPS) for measurement of chemical composition of the treated surfaces. The XPS spectra were acquired on a VG Scientific ESCALAB 250 photoelectron spectrometer with monochromatic Al K $\alpha$ X-rays $(1486.6 \mathrm{eV})$. All spectra were referenced to the $\mathrm{C} 1 \mathrm{~s}$ peak of the aliphatic carbon atoms, which was assigned a value of $284.6 \mathrm{eV} .{ }^{15}$ Spectra were taken at a $45^{\circ}$ electron takeoff angle which roughly corresponds to an analysis depth of $5 \mathrm{~nm}$. 


\subsection{Sample preparation}

Plates of PSMA were made by compression molding at $160^{\circ} \mathrm{C}$. After PET film is plasma treated, the film is immediately placed on a PSMA plate surface and the sample is placed in a compression mold under a slight pressure and annealed to enhance the bonding between PET and PSMA at $150^{\circ} \mathrm{C}$. The bonding is expected to occur by reaction of the amine with an anhydride group on the PSMA to first form an amic acid and followed by ring closure to form an imide shown in Fig. 1. ${ }^{16,17}$ Since the ADCB test requires that the materials on both side of the interface be elastic beams, PET film alone bonded on a PSMA plate is not suitable for the test. The problem was avoided by backing the PET film with a poly (methyl methacrylate) thicker plate (thickness in the range of $1-3 \mathrm{~mm}$ ) and commercial cyanoacrylate glue for bonding them as shown in Fig. 2. After the fracture experiment, the fracture surface is analyzed by using scanning electron microscopy and XPS measurement.

\subsection{Fracture toughness measurement}

The interfacial fracture toughness, or the critical energy release rate, Gc is measured using the asymmetric double cantilever beam (ADCB) method. A schematic drawing of this method is shown in Fig. 2. A razor blade of known thickness $\Delta$ is inserted into the PET/PSMA interface. A crack is initiated ahead of edge of the razor blade. This razor blade is driven by a servo motor at a constant speed $\left(5 \times 10^{-6} \mathrm{~m} / \mathrm{s}\right)$. Steady state crack propagation was established after several minutes. Based on Kanninen's calculation for a small crack in a biomaterial each with finite elasticity, the fracture toughness of

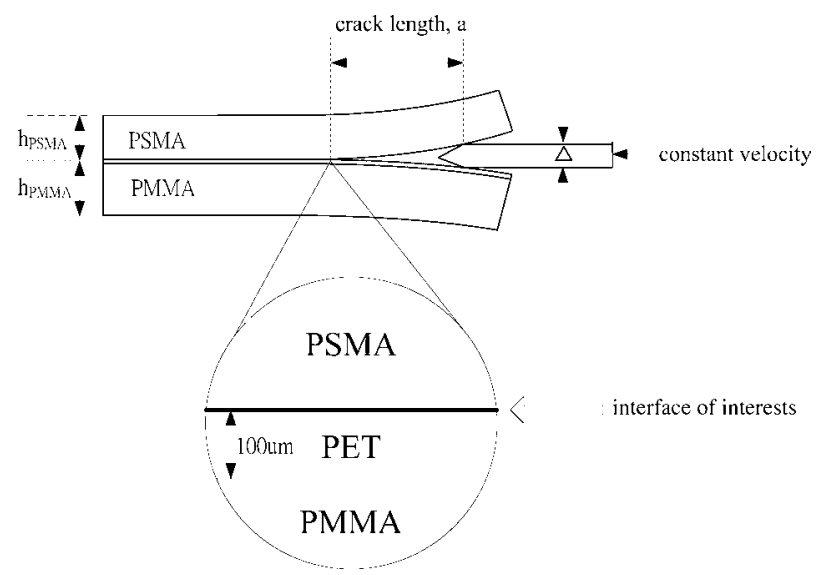

Fig. 2. The schematic drawing of a PET/PSMA sample showing details of the sandwich structure (the enlarged circular drawing) and the asymmetric double cantilever beam method for fracture toughness measurement.

the interface can be measured from the following equation $^{18}$ :

$$
\mathrm{Gc}=\frac{3 \Delta^{2}}{8 a^{4}}\left(E_{1} E_{2} h_{1}^{3} h_{2}^{3}\right) \frac{\left(C_{1}^{2} E_{2} h_{2}^{3}+C_{2}^{2} E_{1} h_{1}^{3}\right)}{\left(C_{1}^{3} E_{2} h_{2}^{3}+C_{2}^{3} E_{1} h_{1}^{3}\right)^{2}},
$$

where $C_{1}=1+0.64 h_{1} / a$ and $C_{2}=1+0.64 h_{2} / a$. $E_{1}$ and $E_{2}$ are the young's moduli and $h_{1}(\sim 1.6 \mathrm{~mm})$ and $h_{2}(2.0 \mathrm{~mm})$ are the thickness of PSMA and PMMA beams, respectively, since the thickness of the backing PMMA beam $(\sim 2 \mathrm{~mm})$ for PET film is much larger than the PET itself $(\sim 100 \mu \mathrm{m})$. It is now known that as a crack propagates along a interface between two materials with different elastic moduli and thicknesses the stress field of the crack tip along the interface has both tensile (mode I) and shear (mode II) components. ${ }^{19}$ The effect of mode I and mode II stress on the fracture energy and location of failure will be discussed in the future publication.

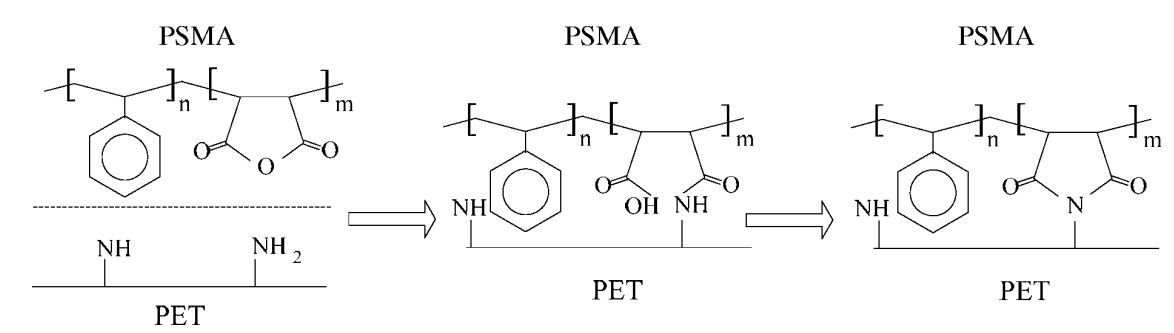

Fig. 1. The reaction between amine groups generated from the nitrogen plasma treatment and anhydride groups from PSMA. 


\section{Results and Discussion}

The wetting properties of PET were examined by water contact angle measurements. As shown in Fig. 3, the equilibrium contact angle on untreated and plasma-treated PET film surface is plotted as a function of treatment time for three different plasma energies of 25, 50 and $75 \mathrm{~W}$ and at a nitrogen pressure of 150 mTorr. For each sample, five measurements on different locations of the sample surface were averaged to form an equilibrium value of the contact angle. With increasing plasma treatment time, the averaged contact angle decreases quickly for all treatment energies. The higher the energy is, the quicker the contact angle decreases. However, the contact angles levels off roughly at the same value for all plasma energies for treatment time $\geq 50 \mathrm{~s}$. The decrease in the contact angle shows that hydrophilic PET surfaces are generated after plasma treatment. ${ }^{20,21}$

Detailed surface elemental compositions of untreated and treated PET film were revealed by XPS survey scan measurements as shown in Fig. 4. PET is initially free of nitrogen. With increasing treatment time, PET shows an increase in the amount of nitrogen incorporation at the surface as seen from the growth of $\mathrm{N}$ 1s peak in Fig. 4. The ratio of the area under the $\mathrm{C} 1$ s peak to that under $\mathrm{N}$ 1s peak, corrected for their ionization cross-sections,

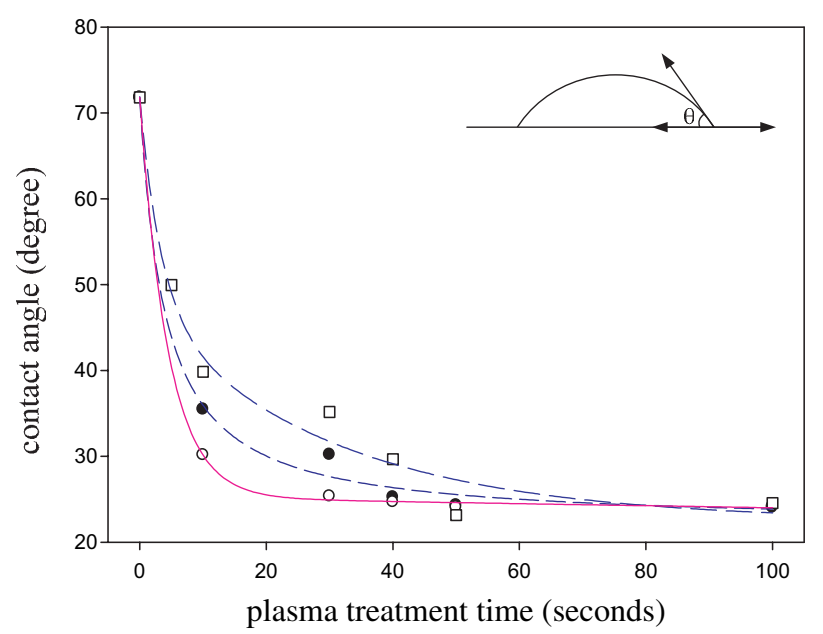

Fig. 3. The contact angle, $\theta$, of PET surface is plotted as a function of plasma treatment time at nitrogen pressure of $150 \mathrm{mT}$ for different output energy of $(\square) 30$, (•) 50 and (o) $70 \mathrm{~W}$.

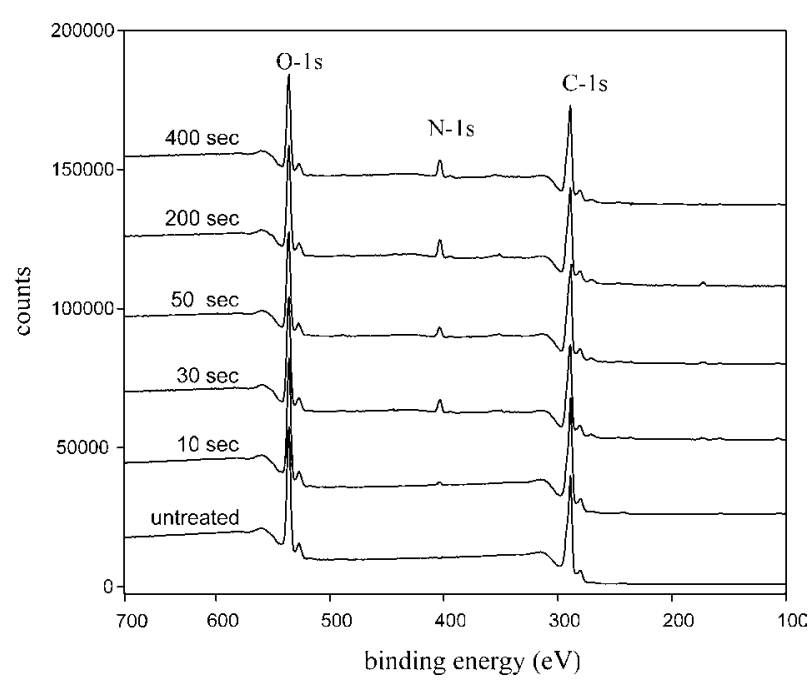

Fig. 4. XPS survey scans for PET treated with 0, 10, 30, 50,200 , and $400 \mathrm{~s}$ of nitrogen plasma at $150 \mathrm{mT}$ pressure and $50 \mathrm{~W}$ output power.

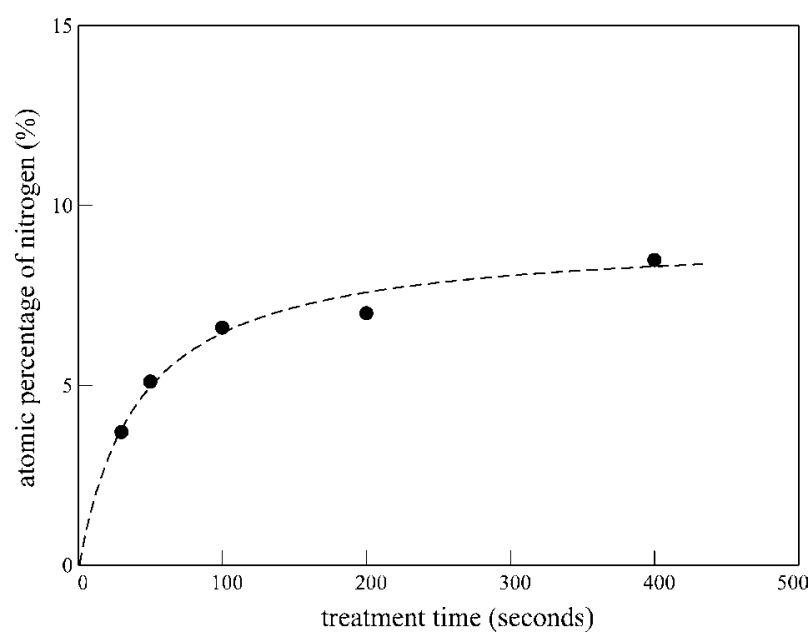

Fig. 5. The atomic percent of incorporated nitrogen measured from XPS as a function of nitrogen plasma treatment time.

provides the relative atomic percentage of the nitrogen incorporation in the treated surfaces.

It is shown from Fig. 5 that with increasing plasma treatment time, the amount of nitrogen incorporation increases quickly within $100 \mathrm{~s}$ of plasma treatment and gradually reaches a saturation level of $\sim 8-8.5 \%$ ( $\mathrm{N} / \mathrm{C}$ in atomic percentage) for treatment time $\geq \sim 100 \mathrm{~s}$. After PET film is plasma-treated, bonding between PET film and PSMA is achieved by annealing the bi-materials at various temperatures 
from $100^{\circ} \mathrm{C}$ to $150^{\circ} \mathrm{C}$ for $2 \mathrm{~h}$. For bi-material samples annealed at a temperature less than $120^{\circ} \mathrm{C}$, the glass transition temperature of PSMA, there was essentially no bonding strength between PET and PSMA and many PET/PSMA sample cracked as soon as it was removed from the compression mold. For samples annealed at a temperature higher than $160^{\circ} \mathrm{C}$, it was found that the PET/PSMA specimen was bent after annealing due to the mismatch of thermal expansion coefficient of each layer in the specimen. In addition, the energy release rate Gc given by Eq. (1) assumes that the ADCB specimen was in a stressfree state before the razor blade was inserted. Therefore, for sample annealed at a temperature $>160^{\circ} \mathrm{C}$, an actuate Gc value cannot be obtained without incorporating additional Gc contribution which takes into account the residual stress from the mismatch of thermal expansion coefficient. ${ }^{22}$ Therefore, the Gc values for high temperature annealing are not reported here.

Figure 6 shows the fracture toughness of interface for plasma-treated PET/PSMA samples annealed at $130^{\circ} \mathrm{C}$ and $150^{\circ} \mathrm{C}$ for $2 \mathrm{~h}$. For samples annealed at $130^{\circ} \mathrm{C}$, the fracture toughness of the interface remains at a low value of $\sim 5-10 \mathrm{~J} / \mathrm{m}^{2}$ for all plasma treatment times. However, when plasma-treated PET/PSMA specimen is annealed at $150^{\circ} \mathrm{C}, 30^{\circ} \mathrm{C}$ above the glass transition temperature of PSMA, for $2 \mathrm{~h}$, the fracture toughness of the interface is

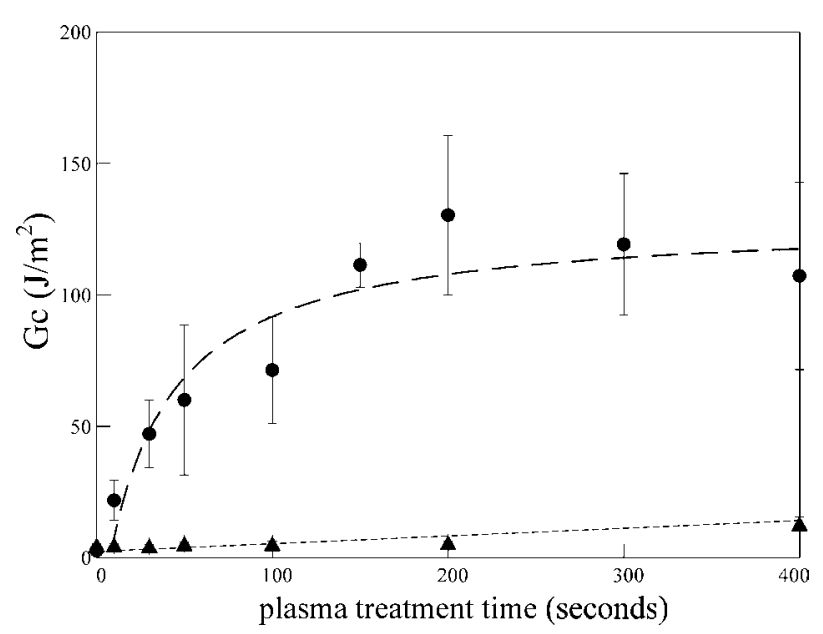

Fig. 6. The fracture toughness of PET/PSMA interface reinforced with plasma treatment at a nitrogen pressure of $150 \mathrm{mT}$ and at $50 \mathrm{~W}$ output energy followed by annealing the biomaterial $(\boldsymbol{\Delta})$ at $130^{\circ} \mathrm{C}$ and $(\bullet)$ at $150^{\circ} \mathrm{C}$ for $2 \mathrm{~h}$. increased with increasing plasma treatment time. The fracture toughness increases quickly within $100 \mathrm{~s}$ of plasma treatment and reaches a maximum plateau Gc value of $100-120 \mathrm{~J} / \mathrm{m}^{2}$ for time $>100 \mathrm{~s}$.

The effects of annealing temperature and plasma treatment on the fracture toughness and its maxima strongly suggest that a reaction takes place between plsma-treated PET surface and PSMA annealed at elevated temperatures above $T_{\mathrm{g}}$ of PSMA and forms chemical bonds that enhance the adhesion of the interface. Particularly, the fracture toughness result follows the same trend as the amount of nitrogen incorporation on PET surface as the plasma treatment time increases. This result indicates that there exists a correlation between the fracture energy and the amount of chain connectors generated from the in situ interfacial reaction between PET and PSMA. The amount of chain connectors which are responsible for the enhancement of the adhesion is normally expressed as areal chain density, $\Sigma$ with a unit of connecting chain per unit area. Such correlation between Gc and $\Sigma$ will be discussed in the future publication.

After samples were fractured, scanning electron microscopy (SEM) was used to examine the failure surface of PET film. Figure 7(a) shows a SEM micrograph of fractured surface on PET side of the test sample plasma treated for $10 \mathrm{~s}$ followed by annealing at $150^{\circ} \mathrm{C}$ for $2 \mathrm{~h}$. The fractured surface appears to be relative flat with no significant plastic deformation. The morphology of fracture surface correlate well with its corresponding Gc value $\left(<20 \mathrm{~J} / \mathrm{m}^{2}\right)$ since the adhesion of the interface is relatively weak. When the nitrogen-containing functional groups on PET surface increases due to the increase of plasma treatment time and the functional groups reacted with PSMA forming more chemical linkages, the stress at the interface was large enough to induce plastic deformation during fracture process.

In treating PET film with nitrogen plasma treatment a variety of carbon-nitrogen functional groups, e.g., amine, amide, imide, etc. can be generated. However, only amine functional groups are important since both imide and amide functional groups do not react with anhydride groups at an appreciable rate under the current annealing temperature range. ${ }^{23-25}$ Based on the high resolution XPS spectra of PET surface treated with plasma, the atomic percentage of amine and amide groups on PET surface 


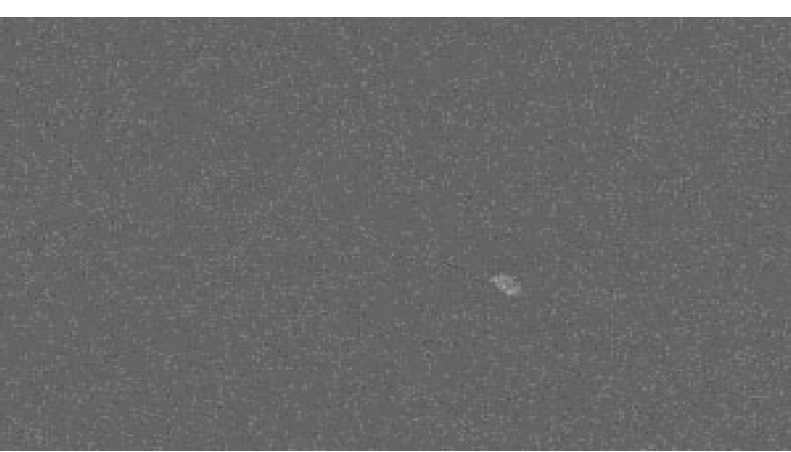

(a)

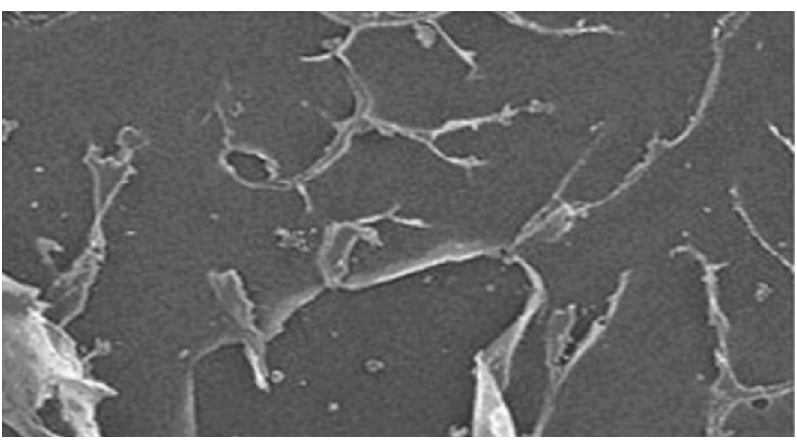

(b)

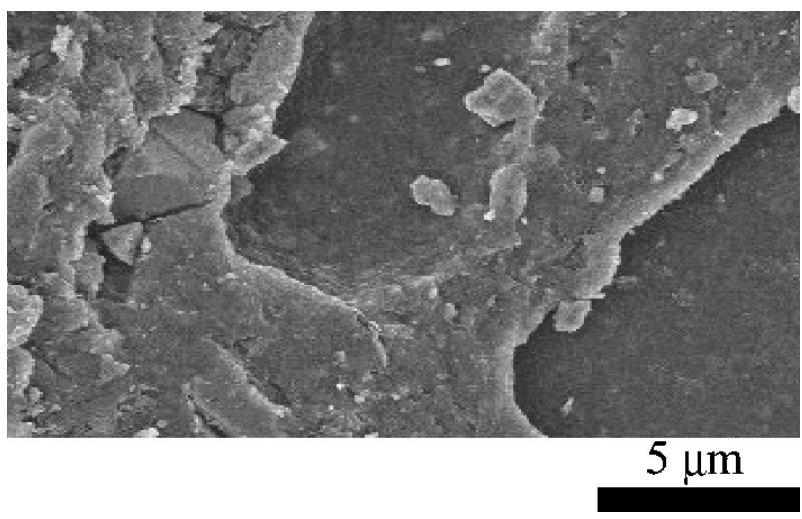

(c)

Fig. 7. Scanning electron micrograph of PET fracture surface: (a) plasma treatment time $10 \mathrm{~s}$, (b) $30 \mathrm{~s}$ and (c) $100 \mathrm{~s}$. The PET surface was previously plasma-treated at nitrogen pressure of $150 \mathrm{mT}$ and $50 \mathrm{~W}$ output energy and subsequently annealed with PSMA at $150^{\circ} \mathrm{C}$ for $2 \mathrm{~h}$.

at different plasma treatment time are tabulated in Table 1. Although the amount of amide functional groups on PET surface grows linearly with increasing plasma treatment time while the fracture toughness increases initially and reach a saturation value for time $>100 \mathrm{~s}$. The above result suggests that amine functional groups react with anhydride groups
Table 1. Temporal chemical composition change of untreated and treated PET film surface for treatment conditions of $150 \mathrm{mT}$ of nitrogen pressure and $50 \mathrm{~W}$ of output power.

Treatment Contribution of C 1s component (\%) time (s)

\begin{tabular}{rccccc}
\hline & $\mathrm{C}-\mathrm{C}$ & $\mathrm{C}-\mathrm{O}$ & $\mathrm{C}=\mathrm{O}$ & $\mathrm{C}-\mathrm{N}$ & $\mathrm{O}=\mathrm{C}-\mathrm{N}$ \\
0 & 63.2 & 22.5 & 14.3 & - & - \\
30 & 55.7 & 26.7 & 13.9 & 2.8 & 0.9 \\
50 & 55.2 & 24.4 & 15.3 & 3.7 & 1.4 \\
100 & 54.2 & 24.1 & 14.9 & 5.2 & 1.6 \\
200 & 53.6 & 23.3 & 15.1 & 6.2 & 1.8 \\
400 & 54.0 & 24.2 & 13.2 & 6.4 & 2.2 \\
\hline
\end{tabular}

to form loading bearing in situ copolymers at the interface that enhance the interface.

Traditionally, the mechanical property of PET film can be measured using simple uniaxial tensile testing. The properties measured from the test are normally related to the in-plane property of the film sample. However, property in the thickness of film specimen cannot be easily measured. Therefore, there are great demands to explore new methods of measuring property in the thickness direction, particularly the fracture property. Since the interlayer fracture toughness depends strongly on the crystallinity, crystal orientation, the amount of tie molecules and their conformation which can be greatly affected by the manufacturing process of PET film. Therefore, with the development of this technique, the effect of process variation on the interface fracture property can be quantitatively measured.

\section{Conclusions}

In this study, we have developed a method to quantitatively measure adhesion of thin film substrate (film thickness $\sim 100 \mu \mathrm{m}$ ) which normally cannot be easily measured by conventional methods, e.g., peel test. We have used nitrogen plasma treatment to enhance the adhesion of biaxially oriented PET film and PSMA bulk materials. The amount of nitrogen incorporation can be increased to a maximum level of roughly 8.5 atom\%. The adhesion of the PET/PSMA follows the similar trend as the incorporated nitrogen amount with increasing plasma treatment time. Two types of failure mechanisms were 
observed, namely, interfacial failure at PET/PSMA interface and failure at the interlayer in PET film. In this study, the interlayer fracture toughness of a biaxially orientated PET film can also be measured with a value of $\sim 110-120 \mathrm{~J} / \mathrm{m}^{2}$.

\section{Acknowledgments}

Financial supports by the National Science Council of Taiwan (NSC92-2216-E-002-015 and NSC93-2120M-002-010) and by US Air force (AFOSR-AOARD04-4023) are greatly appreciated.

\section{References}

1. E. Werner, S. Janocha, M. Hopper and K. J. MacKenzie, Encyclopedia of Polymer Science and Engineering, Vol. 12, ed. J. I. Kroschwlsitz (John Wiley, 1989), p. 193.

2. S. Fakirov, Handbook of Thermoplastic Polyesters (Wiley-VCH, 2005).

3. G. W. Hastings, Cardiovascular Biomaterials (Springer-Verlag, London, 1992).

4. P. A. Ramires, L. Mirenghi, A. R. Romano, F. Palumbo and G. Nicolardi, J. Biomed. Mater. Res. 51 (2000) 535-539.

5. F. R. Pu, R. L. Williams, T. K. Markkula and J. A. Hunt, Biomaterials 23 (2002) 2411.

6. V. B. Gupta and S. Kumar, J. Appl. Polym. Sci. 26 (1981) 1865.

7. D. Bollen, J. Deneir and E. Aernoudt, J. Mater. Sci. 24 (1989) 2957.

8. U. Goschel, Polymer 36 (1995) 1157.

9. H. Yasuda, H. C. Marsh, E. S. Brandt and C. N. Reilley, J. Polym. Sci. Polym. Chem. Ed. 15 (1977) 991.
10. D. Briggs, J. Adhesion 13 (1982) 287.

11. J. M. Pochan, L. J. Gerenser and J. F. Elman, Polymer 27 (1986) 1058.

12. M. Collaud, P. Groening, S. Nowak and L. Schlapbach, J. Adhesion Sci. Technol. 8 (1994) 1115.

13. D. Cohn and T. Stern, Macromolecules 33 (2000) 137-142.

14. M. K. Shi, G. Dunham, M. E. Gross, G. L. Graff and P. M. Marin, J. Adhesion Sci. Technol. 14 (2000) 1485 .

15. G. Beamson and D. Briggs, High Resolution XPS of Organic Polymers (John Wiley \& Sons, 1992).

16. C. Scott and C. W. Macosko, J. Polym. Sci. Polym. Phys. 32 (1994) 205.

17. P. Marechal, G. Coppens, R. Legras and J. Dekoninck, J. Polym. Sci., Part A: Polym. Chem. 33 (1995) 757.

18. M. F. Kanninen, Int. J. Fract. 9 (1973) 83.

19. J. R. Rice, Fracture - An Advanced Treatise, Vol. 2, ed. H. Liebowitz (Academic Press, 1968), p. 191.

20. S. Wu, Polymer Interfaces and Adhesion (Dekker, New York, 1982).

21. K. L. Mittal and A. Pizzi (eds.), Adhesion Promotion Techniques: Technological Applications (Dekker, New York, 1999).

22. Y. Sha, C. Y. Hui, E. J. Kramer, S. F. Hahn and C. A. Berglund, Macromolecules 29 (1996) 4728-4736.

23. E. Boucher, J. P. Folkers, H. Hervet, L. Leger and C. Creton, Macromolecules 29 (1996) 774.

24. E. Boucher, J. P. Folkers, C. Creton, H. Hervet and L. Leger, Macromolecules 30 (1997) 2102-2109.

25. L. J. Gerenser, J. M. Grace, G. Apai and P. M. Thompson, Surf. Interf. Anal. 29 (2000) 12. 
Copyright of Surface Review \& Letters is the property of World Scientific Publishing Company and its content may not be copied or emailed to multiple sites or posted to a listserv without the copyright holder's express written permission. However, users may print, download, or email articles for individual use. 